PUPT-1671

hep-th/9612051

\title{
Fixed scalar greybody factors in five and four dimensions
}

\author{
Igor R. Klebanov and Michael Krasnitz \\ Joseph Henry Laboratories \\ Princeton University \\ Princeton, New Jersey 08544
}

\begin{abstract}
We perform the classical gravity calculations of the fixed scalar absorption cross-sections by $D=5$ black holes with three charges and by $D=4$ black holes with four charges. We obtain analytic results for the cases where the energy and the left and right moving temperatures are sufficiently low but have arbitrary ratios. In $D=5$ the greybody factor is in perfect agreement with the recent calculation performed in the context of the effective string model for black holes. In $D=4$ the formula for the greybody factor in terms of the energy and the temperatures differs from that in $D=5$ only by the overall normalization. This suggests that the fixed scalar coupling to the effective string in $D=4$ is identical to that in $D=5$.
\end{abstract}

December 1996 


\section{The $D=5$ black holes}

\subsection{Introduction}

Recently remarkable progress has been achieved in describing $D=5$ black holes with three different $U(1)$ charges [1, 2, 3, 团 in the string theory context. In the extremal limit these black holes preserve $1 / 8$ of the original supersymmetry and also have a finite horizon area. They may be embedded into string theory using intersecting D-branes, and the resulting entropy of string states agrees with the Bekenstein-Hawking entropy [1, 3, 团. It is believed that their low-energy dynamics is described by small fluctuations of a long intersection string [5]. The model involves $n_{1} 1$-branes marginally bound to $n_{5} 5$-branes, with some longitudinal momentum along the 1-branes carried by left moving open strings. In the near-extremal case, right movers are also present, so that a left moving and a right moving open string may collide to produce an outgoing closed string [3, 6]. The inverse of this process, which gives the leading order contribution to the absorption of closed strings, was also found to be in agreement with the semiclassical gravity, up to an overall normalization [7]. Das and Mathur [8] subsequently normalized the leading emission and absorption rates, both in semiclassical gravity and in the D-brane picture, and found perfect agreement. The specific picture used in [8] follows that suggested in [9, 5]: the low-energy dynamics of the D-brane configuration is captured by a single string with winding number $n_{1} n_{5}$ which is free to vibrate only within the 5-brane hyperplane. The calculation of emission and absorption was generalized to charged particles in [10]. Furthermore, Maldacena and Strominger [11] showed that the agreement between the effective string model and the general relativity continues to hold when the energy, $\omega$, and the left and right moving temperatures, $T_{L}$ and $T_{R}$, are all comparable. In terms of the four radii of the black hole, the parameter region considered in [11] is

$$
r_{0}, r_{K} \ll r_{1}, r_{5}
$$

The greybody factor in this regime has the dependence on $\omega, T_{L}$ and $T_{R}$ which provides strong evidence in favor of the effective string model of $D=5$ black holes [11].

Even more intricate evidence in favor of this model was recently provided by the calculations of the fixed scalar greybody factors [12]. The fixed scalars [13] are the special massless fields whose values on the horizon of an extremal black hole are fixed by the $U(1)$ charges. This fixing translates into a suppression of the absorption and emission at low energies compared to the ordinary massless scalars [14]. In [12] it was shown that the fixed scalars couple to the effective string differently than the ordinary scalars. A specific fixed scalar $\nu$, related to the volume of $T^{4}$ around which the 5-branes are wrapped, was found to couple to the world sheet as

$$
\frac{1}{4 T_{\text {eff }}} \int d^{2} \sigma T_{++} T_{--} \nu
$$

where $T_{\text {eff }}$ is the string tension, while $T_{++}$and $T_{--}$are the left and right moving components of the stress-energy tensor on the effective string. The effective string calculation of the 
absorption cross-section, which utilized the methods of thermal field theory, yielded a simple analytic formula [12],

$$
\sigma_{\mathrm{abs}}=\frac{\kappa_{5}^{2} L_{\mathrm{eff}}}{\left(32 \pi T_{\mathrm{eff}}\right)^{2}}\left(\omega^{2}+16 \pi^{2} T_{L}^{2}\right)\left(\omega^{2}+16 \pi^{2} T_{R}^{2}\right) \omega \frac{e^{\frac{\omega}{T_{H}}}-1}{\left(e^{\frac{\omega}{2 T_{L}}}-1\right)\left(e^{\frac{\omega}{2 T_{R}}}-1\right)}
$$

where $\kappa_{5}$ is the $D=5$ gravitational constant, and $L_{\text {eff }}$ is the length of the effective string, which is related to the radii by

$$
\kappa_{5}^{2} L_{\mathrm{eff}}=4 \pi^{3} r_{1}^{2} r_{5}^{2}
$$

In [12 the greybody factor (3) was shown to agree with the general relativity absorption calculation carried out in the extremal $\left(T_{R}=0\right)$ and the near-extremal $\left(T_{R} \ll T_{L}\right)$ cases. For technical reasons, these calculations were carried out for $r_{1}=r_{5}=R$, and the effective string tension required for the agreement was found to be

$$
T_{\text {eff }}=\frac{1}{2 \pi R^{2}}
$$

Since $R^{2}$ grows as the number of D-branes, this formula confirms the idea that the effective string is fractionated [15, 16].

In this paper we carry out a more general classical absorption calculation, and show its complete agreement with (3). We find it possible to reduce the fixed scalar equation in the inner region (near the horizon) to the hypergeometric equation. After a matching of approximate solutions, the result (3) follows from the well-known asymptotics of the hypergeometric functions.

\subsection{The classical absorption calculation}

The Einstein metric for the five-dimensional black hole is [2, 3, 17, 4]

$$
d s^{2}=-f^{-2 / 3} h d t^{2}+f^{1 / 3}\left(h^{-1} d r^{2}+r^{2} d \Omega_{3}^{2}\right)
$$

where

$$
h(r)=1-\frac{r_{0}^{2}}{r^{2}}, \quad f(r)=\left(1+\frac{r_{K}^{2}}{r^{2}}\right)\left(1+\frac{r_{1}^{2}}{r^{2}}\right)\left(1+\frac{r_{5}^{2}}{r^{2}}\right) .
$$

$r_{0}$ is the non-extremality parameter of the black hole, while $r_{K}, r_{1}, r_{5}$ are related to the other charges. One also introduces the hyperbolic angle $\sigma$ defined by

$$
r_{K}=r_{0} \sinh \sigma
$$

The left and right temperatures are [4, 11]

$$
T_{L}=\frac{r_{0} e^{\sigma}}{2 \pi r_{1} r_{5}}, \quad T_{R}=\frac{r_{0} e^{-\sigma}}{2 \pi r_{1} r_{5}},
$$


and the Hawking temperature is their harmonic average:

$$
\frac{2}{T_{H}}=\frac{1}{T_{L}}+\frac{1}{T_{R}}
$$

We will confine ourselves to the dilute gas region (1) and consider sufficiently low frequencies,

$$
\omega r_{i} \ll 1
$$

This is the parameter region decribed by the effective string model.

The equation describing the propagation of the fixed scalar $\nu$ near a black hole with $r_{1}=r_{5}=R$ (only in this case does it seem possible to obtain a simple equation) was derived in 12 ,

$$
\left[\left(h r^{3} \frac{d}{d r}\right)^{2}+\left(r^{2}+R^{2}\right)^{2}\left(r^{2}+r_{K}^{2}\right) \omega^{2}-\frac{8 r^{4} R^{4}}{\left(r^{2}+R^{2}\right)^{2}} h\right] \nu(r)=0 .
$$

We solve (6) by the matching technique as in [8, 11, 12]. Namely, we divide space into three regions, in each of which the equation simplifies and can be solved explicitly:

I. The Near region: $r \ll R$.

II. The Middle region: $r_{0} \ll r \ll 1 / \omega$.

III. The Far region: $r \gg R$.

Note that, since $r_{0} \ll R \ll 1 / \omega$, the middle region overlaps each of the other two.

Now we show how (6) simplifies in each of the three regions. In regions II and III we may approximate $h(r)=1$. In region III the potential is negligible compared to the frequency term, and the equation reduces to

$$
\left[r^{-3} \frac{d}{d r} r^{3} \frac{d}{d r}+\omega^{2}\right] \nu_{I I I}=0
$$

The solution may be written as

$$
\nu_{I I I}=\alpha \frac{J_{1}(\omega r)}{\omega r}+\beta \frac{N_{1}(\omega r)}{\omega r}
$$

where $J_{1}, N_{1}$ are correspondingly the Bessel and Neumann functions.

In region II the frequency term may be neglected, and we have the approximate equation

$$
\left[\left(r^{3} \frac{d}{d r}\right)^{2}-8 \frac{R^{4}}{H^{2}}\right] \nu_{I I}=0
$$

with

$$
H(r)=1+\frac{R^{2}}{r^{2}}
$$

The solution has two undetermined parameters,

$$
\nu_{I I}=\frac{A}{H(r)}+B H^{2}(r) .
$$


In region I (6) becomes

$$
\left[\left(h r^{3} \frac{d}{d r}\right)^{2}+R^{4}\left(r^{2}+r_{K}^{2}\right) \omega^{2}-8 r^{4} h\right] \nu_{I}=0 .
$$

In terms of the variable $z=1-\frac{r_{0}^{2}}{r^{2}}$ we get

$$
\left[\left(z \frac{d}{d z}\right)^{2}+D+\frac{C}{1-z}-\frac{2 z}{(1-z)^{2}}\right] \nu_{I}=0
$$

where

$$
D=\frac{\omega^{2}\left(T_{L}-T_{R}\right)^{2}}{64 \pi^{2}\left(T_{L} T_{R}\right)^{2}}, \quad C=\frac{\omega^{2}}{16 \pi^{2} T_{L} T_{R}} .
$$

In the immediate vicinity of the horizon, we have $z \ll 1$, and the equation reduces to

$$
\left[\left(z \frac{d}{d z}\right)^{2}+(C+D)\right] \nu_{I}=0
$$

The incoming solution at the horizon is

$$
\nu_{I}(z \rightarrow 0)=e^{-i \sqrt{C+D} \log z}=z^{-i(a+b) / 2},
$$

where following [1] we defined

$$
a=\frac{\omega}{4 \pi T_{L}}, \quad b=\frac{\omega}{4 \pi T_{R}} .
$$

To solve the full region I equation, (17), we will define a new function $F$ by

$$
\nu_{I}(z)=z^{-i(a+b) / 2}(1-z)^{-1} F(z)
$$

Substituting this into (7), we see that that $F$ satisfies a hypergeometric equation.

$$
z(1-z) \frac{d^{2} F}{d z^{2}}+[(1-i a-i b)+(1+i a+i b) z] \frac{d F}{d z}-(1+i a)(1+i b) F=0
$$

In order to get an incoming solution near the horizon, we choose the boundary condition $F(0)=1$. The solution is the hypergeometric function $F(-1-i a,-1-i b, 1-i a-i b ; z)$.

To match this with the region II solution we take the limit $z \rightarrow 1$. Defining $v=1-z$, we expand around $v=0$ :

$$
F(-1-i a,-1-i b, 1-i a-i b ; 1-v)=E+G v+O\left(v^{2}\right)
$$

where

$$
E=\frac{2 \Gamma(1-i a-i b)}{\Gamma(2-i a) \Gamma(2-i b)}
$$


and $G$ is a constant of the same order as $E$ whose value will not be important to us. As a consequence,

$$
\nu_{I}(v \rightarrow 0)=E v^{-1}+G+O(v)=E \frac{r^{2}}{r_{0}^{2}}+G+O(v)
$$

We match this to the small $r$ behavior of the middle region solution,

$$
\nu_{I I}=A \frac{r^{2}}{R^{2}}+B \frac{R^{4}}{r^{4}}
$$

where we have kept the dominant term in the expansion of $H(r)$, by requiring that $\nu$ and its derivative are continuous at $r=r_{m}$. The matching point $r_{m}$ satisfies

$$
r_{0} \ll r_{m} \ll R
$$

Solving the two matching equations, we obtain

$$
A=E \frac{R^{2}}{r_{0}^{2}}+G \frac{2 R^{2}}{3 r_{m}^{2}}, \quad B=G \frac{r_{m}^{4}}{3 R^{4}} .
$$

We see that $B \ll A$. In fact, throughout region II, $B H^{2}$ may be neglected relative to $A H^{-1}$. We can also neglect the second term in $A$ relative to the first one and use $A=E \frac{R^{2}}{r_{0}^{2}}$.

We now match regions II and III. As $\omega r \rightarrow 0$, we have

$$
\nu_{I I I} \rightarrow \frac{\alpha}{2}-\frac{2 \beta}{\pi \omega^{2} r^{2}}
$$

and, as $\frac{R}{r} \rightarrow 0$, we have

$$
\nu_{I I} \rightarrow A\left(1-\frac{R^{2}}{r^{2}}\right)
$$

Matching, we obtain

$$
\alpha=2 A, \quad \beta=\frac{\pi A \omega^{2} R^{2}}{2}
$$

We see that

$$
\frac{\beta}{\alpha} \sim(\omega R)^{2} \ll 1
$$

so that we may neglect $\beta$ in what follows. We obtain

$$
\alpha=2 A=2 E \frac{R^{2}}{r_{0}^{2}} .
$$

The absorption probability is the ratio of the incoming fluxes at the horizon and at infinity. In this case the flux is given by

$$
F=\frac{1}{2 i}\left(\nu^{*} h r^{3} \frac{d}{d r} \nu-\text { c.c. }\right)
$$

and so we get

$$
P_{\text {abs }}=\frac{F_{\text {horizon }}}{F_{\infty}}=\frac{2 \pi}{|\alpha|^{2}} R^{2} \sqrt{r_{0}^{2}+r_{K}^{2}} \omega^{3}
$$


By the Optical Theorem in $D=5$, the absorption cross-section is 8

$$
\sigma_{\mathrm{abs}}=\frac{4 \pi}{\omega^{3}} P_{\mathrm{abs}}
$$

Using the identities

$$
\Gamma(z+1)=z \Gamma(z), \quad|\Gamma(1-i a)|^{2}=\frac{\pi a}{\sinh \pi a}
$$

we obtain the following value for the absorption cross-section,

$$
\sigma_{\mathrm{abs}}=\frac{\pi^{3} R^{8}}{64}\left(\omega^{2}+16 \pi^{2} T_{L}^{2}\right)\left(\omega^{2}+16 \pi^{2} T_{R}^{2}\right) \omega \frac{e^{\frac{\omega}{T_{H}}}-1}{\left(e^{\frac{\omega}{2 T_{L}}}-1\right)\left(e^{\frac{\omega}{2 T_{R}}}-1\right)} .
$$

Using (4) and (5) we find that this is in precise agreement with the absorption cross-section predicted by the effective string model, (3).

\section{The $\mathrm{D}=4$ Case}

\subsection{Introduction}

The $D=4$ black holes with four $U(1)$ charges [19, 20] have many features in common with the $D=5$ black holes considered above. An effective string model for such $D=4$ black holes is motivated by their embedding into M-theory [21, 22]. A specific configuration useful for explaining the Bekenstein-Hawking entropy is the $5 \perp 5 \perp 5$ intersection [22]: there are $n_{1}$ 5-branes in the (12345) hyperplane, $n_{2}$-5-branes in the (12367) hyperplane, and $n_{3}$ 5 -branes in the (14567) hyperplane. One also introduces a left moving momentum along the intersection string (in the $\hat{1}$ direction). If the length of this direction is $L_{1}$, then the momentum is quantized as $2 \pi n_{K} / L_{1}$, so that $n_{K}$ plays the role of the fourth $U(1)$ charge. Upon compactification on $T^{7}$ the metric of the $5 \perp 5 \perp 5$ configuration reduces to that of the $D=4$ black hole with four charges. Just like in the D-brane description of the $D=5$ black hole, the low-energy excitations are signals propagating along the intersection string. In M-theory the relevant states are likely to be small 2-branes with three holes glued into the three different hyperplanes [22]. As a result, the effective length of the intersection string is $L_{\text {eff }}=n_{1} n_{2} n_{3} L_{1}$. This fact, together with the assumption that these modes carry central charge $c=6$, is enough to reproduce the extremal Bekenstein-Hawking entropy, $S=2 \pi \sqrt{n_{1} n_{2} n_{3} n_{K}}$ [22]. In [10] it was shown that this "multiply-wound string" model of the four-charge $D=4$ black hole correctly reproduces the Hawking radiation of both neutral and Kaluza-Klein charged scalars. Furthermore, the ordinary scalar greybody factor agrees with that in the effective string picture of the $D=4$ black holes [18]. The parameter region considered in 18 is:

$$
r_{0}, r_{K} \ll r_{1}, r_{2}, r_{3}
$$


In this section we calculate the fixed scalar greybody factor in this region and show that, up to the overall normalization, it is identical to that in the $D=5$ case. We conclude by discussing possible implications of this equivalence for the coupling of the fixed scalars to the effective string.

\section{The semiclassical gravity analysis}

The metric of the $D=4$ black hole with four charges is 19, 20, 23

$$
d s^{2}=-f^{-1 / 2} h d t^{2}+f^{1 / 2}\left(h^{-1} d r^{2}+r^{2} d \Omega_{2}^{2}\right)
$$

where

$$
h(r)=1-\frac{r_{0}}{r}, \quad f(r)=\left(1+\frac{r_{K}}{r}\right)\left(1+\frac{r_{1}}{r}\right)\left(1+\frac{r_{2}}{r}\right)\left(1+\frac{r_{3}}{r}\right)
$$

Now we define $\sigma$ by

$$
r_{K}=r_{0}(\sinh \sigma)^{2}
$$

The left and right temperatures are [18]

$$
T_{L}=\frac{1}{4 \pi} \sqrt{\frac{r_{0}}{r_{1} r_{2} r_{3}}} e^{\sigma}, \quad T_{R}=\frac{1}{4 \pi} \sqrt{\frac{r_{0}}{r_{1} r_{2} r_{3}}} e^{-\sigma}
$$

and the Hawking temperature is their harmonic average. We will work in the dilute gas region (12) and assume the low energy condition,

$$
\omega r_{i} \ll 1
$$

The equations governing the fixed scalar propagation for this range of parameters were studied in [12]. To get a tractable equation for the fixed scalar fluctuations, we may take three large charges to be equal:

$$
r_{1}=r_{2}=r_{3}=R
$$

Then we have the following equation for the fixed scalar $\nu$,

$$
\left[\left(h r^{2} \frac{d}{d r}\right)^{2}+\omega^{2} r^{4}\left(1+\frac{r_{K}}{r}\right)\left(1+\frac{R}{r}\right)^{3}-2 h R^{2}\left(1+\frac{R}{r}\right)^{-2}\right] \nu=0 .
$$

As before, we solve this by considering the near, middle and far regions defined in the same way as for the $D=5$ case.

In the far region we get the solution

$$
\nu_{I I I}=\alpha \frac{\sin (\omega r)}{\omega r}+\beta \frac{\cos (\omega r)}{\omega r},
$$

while in the middle region the solution is

$$
\nu_{I I}=A\left(1+\frac{R}{r}\right)^{-1}+B\left(1+\frac{R}{r}\right)^{2} .
$$


As before we find that

$$
\beta \ll \alpha, \quad B \ll A,
$$

and this time

$$
A=\alpha .
$$

In the near region we get precisely the same equation as for $D=5$, (7), with $a, b, C, D$ defined through $T_{L}, T_{R}$ as in (8) and (9), and $z=1-\frac{r_{0}}{r}$. Performing the matching in the same way as before, we get

$$
\alpha=\frac{2 R}{r_{0}} \frac{\Gamma(1-i a-i b)}{\Gamma(2-i a) \Gamma(2-i b)} .
$$

It is again convenient to find the absorption probability with the method of fluxes. Now the radial flux is given by

$$
F=\frac{1}{2 i}\left(\nu^{*} h r^{2} \frac{d}{d r} \nu-\text { c.c. }\right) .
$$

The absorption probability is calculated to be

$$
P_{\mathrm{abs}}=\frac{r_{0} \omega^{2}}{\pi T_{H}|\alpha|^{2}} .
$$

By the Optical Theorem,

$$
\sigma_{\mathrm{abs}}=\frac{\pi}{\omega^{2}} P_{\mathrm{abs}}
$$

and we find

$$
\sigma_{\mathrm{abs}}=\pi^{2} R^{7}\left(\omega^{2}+16 \pi^{2} T_{L}^{2}\right)\left(\omega^{2}+16 \pi^{2} T_{R}^{2}\right) \omega \frac{e^{\frac{\omega}{T_{H}}}-1}{\left(e^{\frac{\omega}{2 T_{L}}}-1\right)\left(e^{\frac{\omega}{2 T_{R}}}-1\right)} .
$$

Thus, up to overall normalization, the fixed scalar greybody factor in $D=4$ is identical to that found in $D=5$.

While we do not understand the effective string for the $D=4$ black holes as well as in the $D=5$ case, it is tempting to conjecture that, up to the overall normalization, the coupling of the $D=4$ fixed scalar is given by (2). Then the effective string result for the cross-section is proportional to (3) with $\kappa_{5}$ replaced by $\kappa_{4}$. Comparing this to (14) and using the relation [18]

$$
\kappa_{4}^{2} L_{\text {eff }}=16 \pi^{2} r_{1} r_{2} r_{3}=16 \pi^{2} R^{3}
$$

we find that the effective string tension scales as

$$
T_{\text {eff }} \sim \frac{1}{R^{2}} .
$$

If we denote by $n$ the number of 5-branes in each of the three orientations, we see that $T_{\text {eff }} \sim n^{-2}$ (because the black hole radius scales as $R \sim n$ ). Interestingly, this agrees with the scaling of the string tension necessary to explain the entropy of the near-extremal $5 \perp 5$ configuration [24, 25]. I As was argued in [24], this is precisely the string that arises at the triple intersection of M5-branes.

\footnotetext{
${ }^{1}$ A different interpretation of the rescaling of the string tension was given in [26, 20, 16, 27].
} 


\section{Concluding Remarks}

This paper contributes to a growing list of similarities between $D=5$ black holes with three charges and $D=4$ black holes with four charges. In both cases, the microscopic interpretations of the entropy are provided by effective strings whose physical modes carry central charge $c=6$. Furthermore, the classical gravity calculations of the greybody factors for ordinary massless scalars reveal that they have identical coupling to the effective string 18. Perhaps this was not a surprise since this coupling was given by the minimal twoderivative term. Now we find that even the peculiar fixed scalars have essentially identical greybody factors in $D=5$ and $D=4$. In $D=5$ the greybody factor coincides exactly with that produced by the coupling to $T_{++} T_{--}$on the effective string [12]. This is also the case in $D=4$, although we do not yet know of a derivation of this coupling from an effective string action.2 Based on the accumulating evidence, we feel that one should be able to construct a unified picture of the $D=5$ and $D=4$ black holes which shows that the effective strings responsible for their microscopic degrees of freedom have a common origin.

\section{Acknowledgements}

This article is based on a Princeton University Junior Paper by one of us (M.K.). We are grateful to C.G. Callan, S.S. Gubser and A.A. Tseytlin for useful discussions and comments. The work of I.R. Klebanov was supported in part by DOE grant DE-FG02-91ER40671, the NSF Presidential Young Investigator Award PHY-9157482, and the James S. McDonnell Foundation grant No. 91-48.

\section{References}

[1] A. Strominger and C. Vafa, Phys. Lett B379 (1996) 99, hep-th/9601029.

[2] A.A. Tseytlin, Mod. Phys. Lett. A11 (1996) 689, hep-th/9601177.

[3] C.G. Callan and J.M. Maldacena, Nucl. Phys. B472 (1996) 591, hep-th/9602043.

[4] G. Horowitz, J. Maldacena and A. Strominger, Phys. Lett. B383 (1996) 151, hepth/9603109.

[5] J.M. Maldacena and L. Susskind, Nucl. Phys. B475 (1996) 679, hep-th/9604042.

[6] A. Hashimoto and I.R. Klebanov, Phys. Lett. B381 (1996), hep-th/9604065.

[7] A. Dhar, G. Mandal and S. R. Wadia, Phys. Lett. B388 (1996) 51, hep-th/9605234.

\footnotetext{
${ }^{2}$ We are grateful to A. Tseytlin for many discussions on this point.
} 
[8] S.R. Das and S.D. Mathur, Nucl. Phys. B478 (1996) 561, hep-th/9606185.

[9] S.R. Das and S.D. Mathur, Phys. Lett. B375 (1996) 103, hep-th/9601152.

[10] S.S. Gubser and I.R. Klebanov, hep-th/9608108.

[11] J.M. Maldacena and A. Strominger, "Black Hole Greybody Factors and D-Brane Spectroscopy", hep-th/9609026.

[12] C.G. Callan, Jr., S.S. Gubser, I.R. Klebanov and A.A. Tseytlin, "Absorption of Fixed Scalars and the D-brane Approach to Black Holes", hepth/9610172.

[13] S. Ferrara and R. Kallosh, Phys. Rev D54 (1996) 1514, hep-th/9602136; Phys. Rev. D54 (1996) 1525, hep-th/9603090; S. Ferrara, R. Kallosh, A. Strominger, Phys. Rev. D52 (1995) 5412, hep-th/9508072; G. Gibbons, R. Kallosh and B. Kol, Stanford preprint SU-ITP-96-35, hep-th/9607108.

[14] B. Kol and A. Rajaraman, Stanford preprint SU-ITP-96-38, SLAC-PUB-7262, hepth/9608126.

[15] J. Maldacena, Nucl. Phys. B477 (1996) 168, hep-th/9605016.

[16] E. Halyo, B. Kol, A. Rajaraman and L. Susskind, hep-th/9609075; E. Halyo, hepth/9610068.

[17] M. Cvetič and D. Youm, hep-th/9603100.

[18] S.S. Gubser and I.R. Klebanov, Phys. Rev. Lett. 77 (1996) 4491, hepth/9609076.

[19] M. Cvetič and D. Youm, Phys. Rev. D53 (1996) 584, hep-th/9507090.

[20] M. Cvetič and A. Tseytlin, Phys. Lett. B366 (1996) 95, hep-th/9510097; Phys. Rev. D53 (1996) 5619, hep-th/9512031.

[21] A. Tseytlin, Nucl. Phys. B475 (1996) 149, hep-th/9604035.

[22] I.R. Klebanov and A. Tseytlin, Nucl. Phys. B475 (1996) 179, hep-th/9604166.

[23] M. Cvetič and A. Tseytlin, Nucl. Phys. B478 (1996) 181, hep-th/9606033.

[24] A. Hanany and I.R. Klebanov, hep-th/9606136.

[25] I.R. Klebanov and A.A. Tseytlin, Nucl. Phys. B479 (1996) 319, hep-th/9607107.

[26] F. Larsen and F. Wilczek, Phys. Lett. B375 (1996) 37, hep-th/9511064.

[27] E. Halyo, hep-th/9611175. 